\title{
Bringing science and advocacy together to address health needs of people who inject drugs
}

\author{
Liza Dawson, ${ }^{1}$ Steffanie A Strathdee, ${ }^{2}$ Alex John London, ${ }^{3}$ \\ Kathryn E Lancaster, ${ }_{1}^{4}$ Robert Klitzman, ${ }^{5}$ Irving Hoffman, ${ }^{6}$ Scott Rose, \\ Jeremy Sugarman ${ }^{8}$
}

In crafting our paper on addressing the ethical challenges in HIV prevention research with people who inject drugs (PWID) ${ }^{1}$ we had hoped to stimulate further discussion and deliberation about the topic. We are pleased that three commentaries on our paper have begun this process. ${ }^{2} 34$ The commentaries rightly bring up important issues relating to community engagement and problems in translating research into practice in the fraught environments in which PWID face multiple risks. These risks include acquisition of HIV as well as criminalisation, stigma and lack of access to needed healthcare, prevention and social services. We take this opportunity to respond to the excellent points raised by the commentators.

All of the commentaries support our emphasis on robust community engagement with PWID and other stakeholders in designing and conducting HIV prevention research, but urge us to go farther. Wolfe highlights the difficulty of even engaging with community members in oppressive settings, where authorities severely restrict civil liberties of PWID so that even discussing issues related to drug use and enforcement may place individuals at risk. To overcome such limitations, he appropriately suggests interviewing confidentially those who have previously

\footnotetext{
${ }^{1}$ Division of AIDS, NIH/NIAID, Bethesda, Maryland, USA 2Department of Medicine, University of San Diego, San Diego, California, USA

${ }^{3}$ Department of Philosophy, Carnegie Mellon University, Pittsburgh, Pennsylvania, USA

${ }^{4}$ Department of Epidemiology, Ohio State University, Columbus, Ohio, USA

${ }^{5}$ Department of Psychiatry, Columbia University, New York, USA

${ }^{6}$ Department of Medicine, University of North Carolina, Chapel Hill, North Carolina, USA

${ }^{7}$ Science Facilitation, FHI360, Durham, North Carolina, USA

${ }^{8}$ Berman Institute of Bioethics, Johns Hopkins University, Baltimore, Maryland, USA
}

Correspondence to Dr Liza Dawson, Division of AIDS, NIH/NIAID, Bethesda, MD 20892, USA;

dawson|@niaid.nih.gov been detained in closed settings as part of the community engagement process. Similarly, Wolfe observes that critical issues can be overlooked with a narrow focus on study procedures if contextual factors before, during or after a study are ignored. For example, he cites the risk of overdose for study participants who have been abstinent during a study and subsequently resume injecting when the study concludes. These kinds of risks may not be obvious to researchers, sponsors and oversight bodies who tend to focus primarily on risks during research studies. These insights underscore the need to understand comprehensively the local social and political contexts from the perspectives of PWID themselves. We fully agree that risks at all stages of research, including prestudy and poststudy, are ethically salient and ought not be glossed over in the stakeholder engagement process.

Lavery draws attention to the fact that a wide range of stakeholders have interests that are affected by research. Of note, he highlights how views among certain stakeholders may be incomplete or wrong. Thus, a broader stakeholder engagement process may help identify some of the barriers to translating new research findings to policy, or to using existing evidence-based approaches to healthcare for PWID in settings of oppressive polices.

Haire and Kaldor also push further on community engagement, arguing that communities should have veto power over research they deem unacceptable. Although we are concerned that the language of 'veto power' sets an adversarial tone, the substance of this suggestion is entailed by our position. A primary goal of community engagement should be to determine if a particular study is valuable and appropriate, and, if so, to reach mutually acceptable terms of conducting it. One aim of a set of ethics guidelines, like the seven criteria laid out in our paper, is to establish conditions for ethical acceptability to which stakeholders could reasonably agree. If the ethical criteria cannot be met to the satisfaction of stakeholders representing the interests of PWID, we concur that a particular research initiative should be re-examined and revised to be ethically acceptable.

All of the commentaries are also concerned about the implementation of research findings in settings where instituting evidence-based policies is politically tenuous. For instance, Wolfe cites cases in which research studies had positive results, but were not implemented locally and instead informed regulatory approvals or guidelines in sponsoring countries rather than the countries hosting the research. While such cases are clearly unfortunate, it is difficult to ensure that research in any setting will result in meaningful change in practice. However, as argued in our paper, having locally applicable data available to drive policy change when conditions are favourable can be valuable. The use of research data obtained in a research setting of relative vulnerability to support regulatory approvals in a sponsoring country can raise questions of exploitation; but the prohibition of use of research data to inform approvals anywhere seems antithetical to current efforts to increase transparency and availability of research data more broadly. Nonetheless, exportation of results should not be the primary intent of research.

On the grounds that researchers may not be politically adept and there will be difficulties evaluating the adequacy of these efforts, Haire and Kaldor raise concerns about our criteria regarding the obligation of researchers to engage in policy discussions locally and to advocate globally for PWID to promote the implementation of research findings. In offering these criteria, we do not intend for researchers to transmogrify into politicians. That said, it is relevant to recognise the substantial political abilities researchers often possess in executing any meaningful research project in this context. We believe researchers should contribute to policy discussions by providing data and offering their perspectives and experiences as appropriate. We do not envision a role for research ethics committees or other oversight bodies to adjudicate whether researchers are meeting these responsibilities in the context of individual research studies. At the same time, we believe it is necessary for research sponsors, non-governmental organisations and research institutions to invest in more training for researchers to address implementation challenges and implementation science generally, including the use of diverse methods to 
gather and analyse relevant information, as described by Lavery.

Haire and Kaldor also contend that without requiring stronger guarantees about 'reasonable availability', our requirement that research should fill an evidence gap is incomplete. We are concerned that this point conflates two distinct but related questions. The first concerns whether a study is responsive to the health needs of the host community by investigating a question that fills an evidence gap related to the health of that population. The second concerns arrangements for post-trial access to interventions shown to be safe and effective in a trial. However, as we mention, populations with unmet health needs often live under political conditions that make reasonable availability challenging, and often impossible to guarantee. Our approach is to emphasise the importance of responsiveness to health needs in such cases, along with the additional criteria that we outline, and not to prevent otherwise valuable research from moving forward without an advance political commitment on reasonable availability. The hope is that by stressing the responsiveness requirement and creating the evidence that fills a gap in the host community, research creates the means to improve treatment or prevention practices as soon the political context becomes amenable. We also note that the process of creating the information and interventions necessary to close an evidence gap in a population may itself contribute to positive changes in the political climate, consistent with our recommendation that researchers be more socially active in such contexts. Clearly, an advance commitment for widespread implementation of successful interventions would be preferable, but unfortunately is not often achievable.

On a broader level, Lavery urges the research community, including those working on research ethics, to work harder to gather the kind of evidence needed to understand the process of translating research into policy-even when that evidence is derived from methods deemed less rigorous than conventional clinical trials. Other sources of information, such as qualitative research from stakeholder engagement processes, may provide better evidence of the real problems in addressing health needs of groups like PWID, whose interests are discounted for political, rather than scientific, reasons. Lavery's calls for better implementation science and better implementation research ethics are admirable-but not easy to achieve. Continued efforts by researchers, community advocates and policy makers are essential to unpack this 'wicked problem'-and in some cases, fundamental political change, rather than research, will be needed.

Competing interests None declared.

Provenance and peer review Commissioned; internally peer reviewed.

Author note The views expressed in this article are those of the authors alone, and do not represent any policy or position of the US National Institutes of Health, the US Department of Health and Human Services, or any of its components.

(c) Article author(s) (or their employer(s) unless otherwise stated in the text of the article) 2018. All rights reserved. No commercial use is permitted unless otherwise expressly granted.

A Check for updates

To cite Dawson L, Strathdee SA, London AJ, et al. J Med Ethics 2018;44:165-166.

Received 27 September 2017

Accepted 4 October 2017

Published Online First 27 October 2017

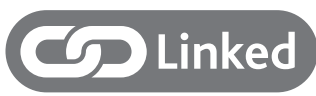

http://dx.doi.org/10.1136/medethics-2015-102895

http://dx.doi.org/10.1136/medethics-2016-103571

- http://dx.doi.org/10.1136/medethics-2016-103572

- http://dx.doi.org/10.1136/medethics-2016-103573

J Med Ethics 2018:44:165-166.

doi:10.1136/medethics-2017-104591

\section{REFERENCES}

1 Dawson L, Strathdee SA, London AJ, et al. Addressing ethical challenges in HIV prevention research with people who inject drugs. J Med Ethics 2018;44:149-58.

2 Haire BG, Kaldor JM. Communities need to be equal partners in determining whether research is acceptable. J Med Ethics 2018;44:159-60.

3 Lavery JV. 'Wicked problems', community engagement and the need for an implementation science for research ethics. J Med Ethics 2018;44:163-4.

4 Wolfe D. Moving the needle: strengthening ethical protections for people who inject drugs in clinical trials. J Med Ethics 2018;44:161-2. 